\title{
Editorial comment on: development and external validation of a model to predict overall survival in patients with resected gallbladder cancer
}

\author{
Mairéad G. McNamara^ \\ Division of Cancer Sciences, University of Manchester \& The Christie NHS Foundation Trust, Manchester, UK \\ Correspondence to: Dr. Mairéad G. McNamara. Division of Cancer Sciences, University of Manchester \& Department of Medical Oncology, The \\ Christie NHS Foundation Trust, Manchester M20 4BX, UK. Email: mairead.mcnamara@nhs.net. \\ Comment on: de Savornin Lohman EAJ, de Bitter TJJ, Hannink G, et al. Development and external validation of a model to predict overall survival in \\ patients with resected gallbladder cancer. Annals of Surg 2021. [Epub ahead of print]. doi: 10.1097/SLA.0000000000005154.
}

Submitted Dec 14, 2021. Accepted for publication Dec 28, 2021.

doi: 10.21037/hbsn-2021-31

View this article at: https://dx.doi.org/10.21037/hbsn-2021-31

The incidence of biliary tract cancer (BTC) (1), including gallbladder cancer (GBC) (2), is increasing. Potentially curative surgery is possible in only approximately $20 \%$, and adjuvant capecitabine, versus observation after resection, has been reported to improve survival in a prespecified sensitivity and per-protocol analyses of the phase 3 randomised trial BILCAP (capecitabine compared with observation in resected BTC) (adjusting for minimisation factors, nodal status, grade, and gender) $(3,4)$. The prognosis for patients with advanced BTC is poor; the median overall survival (OS) for patients receiving standard of care cisplatin/gemcitabine in the first-line setting was 11.7 months in the Advanced Biliary Cancer-02 (ABC-02) study (5). There has been no alteration in the established choice of first-line systemic treatment for advanced BTC for over a decade. However, a recent press release announced that the phase 3 randomised study of durvalumab in combination with cisplatin/gemcitabine versus placebo with cisplatin/gemcitabine as a first-line treatment for patients with advanced BTC (TOPAZ-1) had met its primary endpoint of OS at the interim analysis; detailed results are awaited (NCT03875235).

The benefit from chemotherapy for patients with advanced GBC may differ from other BTC primary sites (6). In addition, a systematic review and meta-analysis exploring the outcomes of palliative systemic chemotherapy in patients with advanced GBC, reported a pooled weighted mean progression-free survival and OS of 4.8 months and 8.3 months, respectively (7). Novel therapeutics are thus required for patients in this BTC subgroup.

Despite the promise of targeted therapy in patients with advanced cholangiocarcinoma (ivosidenib in isocitrate dehydrogenase 1 (IDH1)-mutated cholangiocarcinoma (8) and fibroblast growth factor receptor (FGFR) inhibitors in those with FGFR2 fusions or rearrangements $(9,10)$, similar targeted options for patients with advanced GBC are limited. Interestingly, the multicentre, phase $2 \mathrm{a}$, multiple basket study of pertuzumab and trastuzumab for Human Epidermal growth factor Receptor 2 (HER2)-positive, previously treated metastatic BTC (MyPathway) included 16 of 39 patients (41\%) with GBC; an objective response rate of $23 \%$ was reported (11), and this study provides evidence to support a randomised trial of these agents in patients with BTC, including GBC.

Thus, the ability to predict OS in patients with nonmetastatic, resected GBC could potentially guide which patients have a higher risk of recurrence, and thus may benefit more from adjuvant treatment, and potentially from shorter surveillance imaging intervals thereafter. In the study by de Savornin Lohman et al. (12), the authors propose a model for the prediction of OS in 446 patients $\{380$ patients in a development cohort [nationwide network

^ ORCID: 0000-0002-2272-3678. 
and registry of histo- and cytopathology in the Netherlands (PALGA) and the Netherlands Cancer Registry (NCR)] and 66 in a validation cohort (Australian single institute cohort)\} with resected GBC. All histopathological samples, including those in the validation cohort, were reviewed by an expert pathology team. There was a long median follow-up of 75 months. The final model included 6 variables: age, T-stage, lymph node status, resection margin, differentiation grade and venous invasion. The model demonstrated good discriminatory capacity, reasonable calibration and was reported to outperform the American Joint Committee on Cancer (AJCC) staging system. However, as the authors mention, for the model to perform optimally, all of the included variables are required, and thus it may not be as applicable to those patients who have a GBC diagnosed incidentally, where lymph nodes or liver tissue are not sent for histopathological examination.

Patients included in the development and validation cohorts were diagnosed up to 2017 and 2018 respectively. Only 1 patient received adjuvant chemotherapy (gemcitabine). The median OS was 22 months and 24 months in the development and validation cohorts respectively, which is numerically less than the median OS reported in the BILCAP study (which recruited patients up to 2014) in those patients with BTC who had observation alone post surgery [36 months $(95 \%$ confidence interval: 30-44 months) in the prespecified per-protocol analysis] (3). This may be a reflection of the patients seen in real-world clinical practice, and that only patients with resected GBC were included in the former study (12), with potentially worse prognosis than other BTC primary sites (6). Only $18 \%$ of patients in the observation arm of the BILCAP trial had resected muscle-invasive GBC (3).

However, it does advocate for adjuvant therapy in patients with resected GBC, particularly as de Savornin Lohman et al. (12) reported that only approximately $10 \%$ of patients with resected GBC have a predicted 5-year survival of $>75 \%$ (who may not benefit from the adjuvant approach). The median OS in the capecitibine group in the prespecified per-protocol analysis of BILCAP was 53 months (3), and thus provided a 17 month advantage over observation alone, which is more than the median survival reported for those patients with GBC receiving palliative treatment in the first-line advanced setting (approximately 8.3 months) (7), and therefore decreasing the risk of recurrence in these patients is vital.

A limitation of the model proposed by de Savornin
Lohman et al. (12) may be the lack of inclusion of other potential prognostic factors such as patient performance status, or baseline haematological/biochemicals factors, which have been shown to be prognostic for outcomes in patients with advanced BTC (13).

The authors (12) acknowledge that there are other models in the literature exploring this concept. An externally validated robust model is imperative so that individualised risks for patients with resected GBC can be communicated. An exemplar is the 21-gene expression assay in resected breast cancer, which was evaluated prospectively in a uniformly treated population, and so provided the highest level of evidence supporting the clinical validity and usefulness of an identifed biomarker (14). Adjuvant therapy was recommended based on the clinicopathological features included in the score (which indicated the risk of breast cancer recurrence) (14).

Despite the availability of a number of proposed models for survival prediction in resected GBC, they remain underutilised, and so the potential impact on care outcomes has not been realised; Sharma et al hypothesise that the failure of adoption of clinical risk prediction tools may be due to a lack of standardised integration with electronic health records, and encourages engagement by health care professionals who can provide clinical context to the workflow that a risk prediction model is intending to influence (15).

In conclusion, further exploration of the suggested GBC prediction model in prospective validation clinical trials would be warranted, as informing patients of their predicted survival, as well as sparing them from the potential toxicities of adjuvant chemotherapy, would undoubtedly be favourable.

\section{Acknowledgments}

Funding: None.

\section{Footnote}

Provenance and Peer Review: This article was commissioned by the editorial office, Hepatobiliary Surgery and Nutrition. The article did not undergo external peer review.

Conflicts of Interest: The author has completed the ICMJE uniform disclosure form (available at https://hbsn. amegroups.com/article/view/10.21037/hbsn-2021-31/coif). 
The author has no conflicts of interest to declare.

Ethical Statement: The author is accountable for all aspects of the work in ensuring that questions related to the accuracy or integrity of any part of the work are appropriately investigated and resolved.

Open Access Statement: This is an Open Access article distributed in accordance with the Creative Commons Attribution-NonCommercial-NoDerivs 4.0 International License (CC BY-NC-ND 4.0), which permits the noncommercial replication and distribution of the article with the strict proviso that no changes or edits are made and the original work is properly cited (including links to both the formal publication through the relevant DOI and the license). See: https://creativecommons.org/licenses/by-nc-nd/4.0/.

\section{References}

1. Rahib L, Wehner MR, Matrisian LM, et al. Estimated Projection of US Cancer Incidence and Death to 2040. JAMA Netw Open 2021;4:e214708.

2. Dyba T, Randi G, Bray F, et al. The European cancer burden in 2020: Incidence and mortality estimates for 40 countries and 25 major cancers. Eur J Cancer 2021;157:308-47.

3. Primrose JN, Fox RP, Palmer DH, et al. Capecitabine compared with observation in resected biliary tract cancer (BILCAP): a randomised, controlled, multicentre, phase 3 study. Lancet Oncol 2019;20:663-73.

4. Shroff RT, Kennedy EB, Bachini M, et al. Adjuvant Therapy for Resected Biliary Tract Cancer: ASCO Clinical Practice Guideline. J Clin Oncol 2019;37:1015-27.

5. Valle J, Wasan H, Palmer DH, et al. Cisplatin plus gemcitabine versus gemcitabine for biliary tract cancer. N Engl J Med 2010;362:1273-81.

6. McNamara MG, Lopes A, Wasan H, et al. Landmark survival analysis and impact of anatomic site of origin in

Cite this article as: McNamara MG. Editorial comment on: development and external validation of a model to predict overall survival in patients with resected gallbladder cancer. HepatoBiliary Surg Nutr 2022;11(1):147-149. doi: 10.21037/ hbsn-2021-31 prospective clinical trials of biliary tract cancer. J Hepatol 2020;73:1109-17.

7. Azizi AA, Lamarca A, McNamara MG, et al. Chemotherapy for advanced gallbladder cancer (GBC): A systematic review and meta-analysis. Crit Rev Oncol Hematol 2021;163:103328.

8. Zhu AX, Macarulla T, Javle MM, et al. Final Overall Survival Efficacy Results of Ivosidenib for Patients With Advanced Cholangiocarcinoma With IDH1 Mutation: The Phase 3 Randomized Clinical ClarIDHy Trial. JAMA Oncol 2021;7:1669-77.

9. Abou-Alfa GK, Sahai V, Hollebecque A, et al. Pemigatinib for previously treated, locally advanced or metastatic cholangiocarcinoma: a multicentre, open-label, phase 2 study. Lancet Oncol 2020;21:671-84.

10. Javle M, Lowery M, Shroff RT, et al. Phase II Study of BGJ398 in Patients With FGFR-Altered Advanced Cholangiocarcinoma. J Clin Oncol 2018;36:276-82.

11. Javle M, Borad MJ, Azad NS, et al. Pertuzumab and trastuzumab for HER2-positive, metastatic biliary tract cancer (MyPathway): a multicentre, open-label, phase 2a, multiple basket study. Lancet Oncol 2021;22:1290-300.

12. de Savornin Lohman EAJ, de Bitter TJJ, Hannink G, et al. Development and external validation of a model to predict overall survival in patients with resected gallbladder cancer. Annals of Surg 2021. [Epub ahead of print]. doi: 10.1097/ SLA.0000000000005154.

13. Bridgewater J, Lopes A, Wasan H, et al. Prognostic factors for progression-free and overall survival in advanced biliary tract cancer. Ann Oncol 2016;27:134-40.

14. Sparano JA, Gray RJ, Makower DF, et al. Prospective Validation of a 21-Gene Expression Assay in Breast Cancer. N Engl J Med 2015;373:2005-14.

15. Sharma V, Ali I, van der Veer S, et al. Adoption of clinical risk prediction tools is limited by a lack of integration with electronic health records. BMJ Health Care Inform 2021;28:e100253. 